\title{
Una ópera para la escuela de la ciudadanía ${ }^{1}$
}

\section{An Opera for the School of Citizenship}

Rosa Soliveres Buigues (1) https://orcid.org/0000-0001-6968-8972

José Luis Parejo (2) https://orcid.org/0000-0002-1081-3529

Andrea Giraldez-Hayes (3) https://orcid.org/0000-0002-4374-5200

(1) Universidad Nacional Autónoma de México

(2) Universidad de Valladolid

(3) University of East London

(Recibido: 24 de diciembre de 2019; Aceptado para su publicación: 19 de mayo de 2020)

Cómo citar: Soliveres, R., Parejo, J. L. y Giraldez-Hayes, A. (2021). Una ópera para la escuela de la ciudadanía. Revista Electrónica de Investigación Educativa, 23, e16, 1-13. https://doi.org/10.24320/redie.2021.23.e16.3722

\section{Resumen}

El artículo presenta los resultados preliminares de la investigación llevada a cabo a través del proyecto La Ópera un Vehículo de Aprendizaje (LÓVA) en un grupo de cuarto de Primaria en la Ciudad de México durante el ciclo escolar 2017-2018. El trabajo se encuadra epistemológicamente dentro del paradigma interpretativo y a nivel metodológico en el estudio de caso. El objetivo fue analizar y comprender los procesos de conformación de la ciudadanía con la mediación de la educación musical; en este sentido, el proceso de creación, desarrollo y estreno de una ópera original por parte del alumnado contribuyó al conocimiento de sus derechos y responsabilidades, compromiso y participación social, y reconocimiento de la diversidad.

Palabras clave: educación musical, educación ciudadana, desarrollo social

\section{Abstract}

This article introduces the preliminary results of research conducted within the context of a project entitled "Opera as a Vehicle for Learning" (LÓVA), carried out with a group of fourth-grade elementary students in Mexico City during the 2017-2018 school year. The study is framed epistemologically within the interpretive paradigm and employs a case study methodology. The objective was to explore and understand the processes whereby citizenship is informed by musical education; in this respect, the students' creation, development, and performance of an original work of opera fostered their awareness of their rights and responsibilities, social engagement and commitment, and recognition of diversity.

Keywords: music education, citizenship education, social development

\footnotetext{
${ }^{1}$ El artículo es producto del proyecto <em>Ecologías del aprendizaje en contextos múltiples: análisis de proyectos de educación expandida y conformación de ciudadanía</em> (EDU2014-51961-P), financiado por el Ministerio de Economía y Competitividad, y la beca de movilidad en el extranjero para jóvenes doctores "José Castillejo", realizada en el Institute of Education de la University College London (enero-julio 2019) por el tercer autor del artículo, con el financiamiento del Ministerio de Ciencia, Innovación y Universidades de España.
} 


\section{Introducción}

La Ópera, un Vehículo de Aprendizaje (LÓVA), cuyos antecedentes se pueden encontrar en el proyecto Creating Original Opera (COO), tiene como propósito principal llevar la creación de una ópera original al contexto escolar, de manera que quede incluida en el programa de estudios del aula y se desarrolle a lo largo de todo un curso escolar (Sarmiento, 2012). En algunos proyectos educativos que persiguen acercar la ópera al público más joven prevalece la convivencia de música, teatro, guion literario, plástica y escenografía, entre otras disciplinas, de manera que la ópera pueda ser experimentada prácticamente por todos los alumnos (Faust, 2010). Algunas investigaciones realizadas sobre la implementación de programas de ópera en el entorno escolar concluyen que alentar a los niños a participar en este tipo de programas contribuye a que se involucren en las artes escénicas y en la música más allá del entorno escolar (Dullea, 2017). En la figura 1 se detallan las fases y subfases del proyecto LÓVA que se desarrollaron a lo largo del curso escolar. Durante el proceso de organización y creación de una ópera original se trabajaron, de modo transversal, temáticas en torno a la conformación de una ciudadanía participativa, crítica y socialmente responsable.

Figura 1. Organización del proyecto LÓVA en el Instituto Luis Vives

\begin{tabular}{|c|c|c|c|c|}
\hline $\begin{array}{c}\text { FASE } 1 . \\
\text { PRESENTACIÓN } \\
\text { Septiembre }\end{array}$ & $\begin{array}{l}1.1 \text { Subfase: } \\
\text { Elección del } \\
\text { nombre de la } \\
\text { compañía y } \\
\text { equipo }\end{array}$ & $\begin{array}{l}\text { 1.2 Subfase: } \\
\text { Elección del } \\
\text { logotipo de la } \\
\text { compañía }\end{array}$ & $\begin{array}{l}\text { 1.3 Subfase: El } \\
\text { círculo de la } \\
\text { comunicación }\end{array}$ & \\
\hline $\begin{array}{c}\text { FASE } 2 . \\
\text { METÁFORAS } \\
\text { Octubre }\end{array}$ & $\begin{array}{l}2.1 \text { Subfase: } \\
\text { Lectura de } \\
\text { leyendas y } \\
\text { juegos } \\
\text { colaborativos }\end{array}$ & $\begin{array}{l}\text { 2.2 Subfase: } \\
\text { Diario de las } \\
\text { emociones }\end{array}$ & $\begin{array}{l}2.3 \text { Subfase: } \\
\text { Asignación de } \\
\text { roles para el } \\
\text { liderazgo grupal }\end{array}$ & $\begin{array}{c}\text { 2.4 Subfase: } \\
\text { Interaccionismo } \\
\text { simbólico: qué valor } \\
\text { emocional se atribuye a } \\
\text { un objeto }\end{array}$ \\
\hline $\begin{array}{c}\text { FASE 3. INTRODUCCIÓN } \\
\text { A LA ÓPERA } \\
\text { Noviembre }\end{array}$ & $\begin{array}{l}\text { 3.1 Subfase: } \\
\text { Profesiones de la } \\
\text { compañía }\end{array}$ & $\begin{array}{l}\text { 3.2 Subfase: } \\
\text { Elección del tema, } \\
\text { tesis y mensaje de } \\
\text { la ópera }\end{array}$ & & \\
\hline $\begin{array}{l}\text { FASE 4. ASIGNACIÓN } \\
\text { DE PROFESIONES } \\
\text { Diciembre-Enero }\end{array}$ & $\begin{array}{l}4.1 \text { Subfase: } \\
\text { Redacción de la } \\
\text { historia }\end{array}$ & $\begin{array}{l}4.2 \text { Subfase: } \\
\text { Contextualización } \\
\text { histórica y geográfica } \\
\text { de la ópera }\end{array}$ & $\begin{array}{l}\text { 4.3 Subfase: } \\
\text { Creación de } \\
\text { personajes } \\
\text { originales }\end{array}$ & \\
\hline $\begin{array}{c}\text { FASE 5. ORGANIZACIÓN } \\
\text { DE LA COMPAÑÍA } \\
\text { Febrero-Abril }\end{array}$ & $\begin{array}{l}\text { 5.1 Subfase: } \\
\text { Talleres de } \\
\text { profesiones } \\
\text { (escenógrafos, } \\
\text { intérpretes...) }\end{array}$ & $\begin{array}{l}\text { 5.2 Subfase: } \\
\text { Informe de } \\
\text { progreso de cada } \\
\text { equipo } \\
\text { profesional }\end{array}$ & & \\
\hline $\begin{array}{c}\text { FASE } 6 . \\
\text { REPRESENTACIÓN Y } \\
\text { EVALUACIÓN } \\
\text { Mayo-Junio }\end{array}$ & $\begin{array}{l}6.1 \text { Subfase: } \\
\text { Integración de } \\
\text { las actividades } \\
\text { realizadas en los } \\
\text { equipos }\end{array}$ & $\begin{array}{c}\text { 6.2 Subfase: } \\
\text { Ensayos } \\
\text { generales de la } \\
\text { ópera }\end{array}$ & $\begin{array}{l}6.3 \text { Subfase: } \\
\text { Preestreno y } \\
\text { estreno de la } \\
\text { ópera }\end{array}$ & $\begin{array}{l}\text { 6.4 Subfase: } \\
\text { Exposición } \\
\text { documental y } \\
\text { evaluación del } \\
\text { proyecto }\end{array}$ \\
\hline
\end{tabular}

Además, LÓVA se perfila como una herramienta poderosa que involucra las artes para el desarrollo de la ciudadanía democrática en el alumnado. El informe que Delors (1996) presentó a la UNESCO apostaba por convertir las escuelas en espacios de ciudadanía destinados para aprender a vivir juntos. Resulta necesario que el descubrimiento del otro se convierta en el conocimiento de uno mismo, además de enseñar el respeto a la diversidad para tomar conciencia de posibles semejanzas entre todos. Así, el alumnado podrá tener una visión del mundo completa y al mismo tiempo será capaz de descubrir quién es. La educación para la ciudadanía en las escuelas debe enseñar a los alumnos a comportarse de manera respetuosa con los demás y sus comunidades (Westheimer, 2020). 
Retomando la idea del descubrimiento de la diferencia, conviene advertir que la convivencia no sólo se trata de tolerancia, sino también de reconocimiento de la otredad, esto es, de los valores asociados con el aprendizaje de la diversidad cultural y social, manteniendo la actitud reflexiva y el compromiso social (Starkey, 2017). Giroux (2013) insiste en reconocer el poder de la educación en la construcción de la cultura formadora para, de este modo, luchar por esas esferas públicas, ideales, valores y cursos de acción que ofrecen modos de identidad, relaciones sociales y políticas alternativas. Bokona, directora general de la UNESCO, enfatiza el papel crucial de la educación para la consecución de los Objetivos de Desarrollo Sostenible (ODS) (ONU, 2015).

Durante el desarrollo del proyecto LÓVA se siguieron los principios de la educación para la consecución de los ODS en los que se propone empoderar a los individuos para que reflexionen sobre sus propias acciones sin perder de vista los efectos sociales, culturales, ambientales y económicos que pueden tener dichas acciones. Igualmente se requiere de algunas competencias transversales para alcanzar las metas propuestas por la ONU y conseguir ciudadanos que participen y construyan responsablemente el mundo de hoy.

En la discusión sobre la educación para la ciudadanía, Westheimer (2020, p. 8-9) propone tres modelos del buen ciudadano: "a) The personally Responsible Citizen, b) The Participatory Citizen, y c) The Social Justice Oriented Citizen". El proyecto LÓVA se sitúa en la consecución de ciudadanos personalmente responsables y, a su vez, participativos. En este contexto, la escuela debe aprovechar la potencialidad de la educación artística dado que posibilita el trabajo colaborativo y la resolución de conflictos al promover el entendimiento intercultural y la convivencia con el otro (Cabedo-Mas et al., 2017). En nuestros días resulta evidente que, ya sea por movimientos migratorios de la población, por diversidad étnica o por la realidad que genera la propia idea de sociedad globalizada, el multiculturalismo es un hecho en las aulas. Por consiguiente, la categoría de ciudadanía debe ser considerada a la luz de la diversidad étnica y cultural (Saint Paul, 2011). Las sociedades, antes consideradas como homogéneas, se develan ahora diversas tanto en lo cultural como en lo étnico y racial, al encontrar mayor diversidad en los núcleos de población. En muchos aspectos resulta un desafío para el docente atender a un alumnado cada vez más complejo en su composición e intereses, no sólo en un sentido amplio sino también en lo que se refiere al desarrollo de la ciudadanía democrática y sostenible arriba mencionado.

Tonucci (2017) nos recuerda que la escuela debe convertirse en el espacio en el que los niños puedan expresar su voz. Para ello es relevante ofrecer variedad de actividades, además de conseguir que el alumno pueda expresarse libremente y favorecer su desarrollo. En el caso de México, durante las dos últimas décadas se han llevado a cabo varios estudios acerca de la participación social en las escuelas y la educación para la democracia (Acevedo y López, 2012; Flores-Crespo y Ramírez, 2015; Nieto y Bickmore, 2016). Estos estudios han tomado en cuenta los tres niveles básicos de la educación correspondientes a las tres modalidades escolares: general, indígena y comunitaria (Zurita, 2010). En lo que se refiere a participación social, las conclusiones a las que llegan estos estudios no son nada alentadoras ya que la comunidad escolar continúa sin ser consciente de la utilidad e importancia de su participación. Tanto el alumnado como sus familias sienten que cuando se les invita a participar su opinión no es tenida en cuenta o, sencillamente, no reciben respuestas a sus inquietudes. La consecuencia directa es la progresiva tendencia de la comunidad escolar a distanciarse de la participación social (Baltazar y Saarikallio, 2017; Groarke y Hogan, 2015).

Como contrapeso a estas conclusiones, gran parte del alumnado mexicano, según otros estudios realizados sobre el estado de la educación cívica y ética (Banco Interamericano de Desarrollo, 2011; Cox, 2010), manifiesta estar a favor de la libertad de expresión, el fomento a la igualdad de género y las oportunidades y derechos de todos los grupos raciales y étnicos del país. Todo ello sucede en un ámbito de diversidad lingüística que define al país y que orilla a la coexistencia de ciudadanías paralelas que surgen de dos tradiciones culturales distintas, la europea y la mesoamericana (Díaz, 2015).

\section{Método}

El paradigma de investigación interpretativo utilizado en el presente trabajo busca aumentar la comprensión del contexto del grupo que es objeto de estudio y de los valores de la educación (Higgins, 2015). El objetivo general consiste en analizar y comprender los procesos de conformación de la ciudadanía 
con la mediación de LÓVA. Se opta por el estudio de caso que propone Stake (2005), puesto que persigue la comprensión profunda de la realidad de un caso concreto con la posición de investigadora profesora. En cuanto al diseño del estudio de caso, nuestra investigación responde al tipo instrumental. El grupo de alumnos fue escogido ad hoc por tratarse de una población heterogénea tanto en lo que respecta al nivel académico como en la diversidad de personalidades y edades.

Las técnicas empleadas para la obtención y recogida de datos incluyeron la observación participante compilada en el diario de la investigadora, en donde se relatan a detalle las sesiones sostenidas con la compañía de ópera, así como los avances y logros que se obtuvieron día a día. De igual forma, se recurrió a la evaluación del estado y desarrollo social del alumnado, tarea llevada a cabo por dos maestras que tomaron en cuenta las características del grupo al inicio del curso escolar y su evaluación a lo largo de la implementación del proyecto. De manera especial se consideraron las producciones de aprendizaje del alumnado, testimonios en los que se recabaron los relatos escritos sobre las emociones que les provocaba la asunción de retos, videos y pruebas fotográficas que mostraban lo que para los niños significaba su pertenencia al proyecto LÓVA, así como las declaraciones de satisfacción por el trabajo colaborativo realizado. Por último, al finalizar el curso escolar se realizó una entrevista grupal abierta a las familias, método más flexible para la obtención de datos fuera del aula (Bohnsack, 2004); así se obtuvo la perspectiva de las familias sobre la experiencia del proceso de aprendizaje y desarrollo del proyecto (Dullea, 2019). Estas entrevistas aportaron, de manera narrativa, datos sobre el desarrollo social e individual de cada miembro de la compañía de ópera.

Respecto al análisis de la información, se tomaron en cuenta las primeras impresiones relatadas en el diario de la investigadora, así como los informes de investigación redactados por etapas. De este modo, para el informe final, después de la selección y triangulación de datos, se obtuvieron categorías y subcategorías que permitieron codificar la información y facilitar el análisis, siguiendo el objetivo principal de la investigación (Simons, 2011).

Las categorías empleadas fueron: derechos y responsabilidades, compromiso y participación social, y respeto y valoración de la diversidad. Para el análisis y discusión de los resultados obtenidos sobre el desarrollo de la competencia ciudadana de los miembros del grupo se ideó el siguiente sistema de codificación de los datos (Tabla 1).

Tabla 1. Codificación de datos

\begin{tabular}{|c|c|c|}
\hline Tipo de documento & Codificación & Ejemplo \\
\hline $\begin{array}{l}\text { Diario de la } \\
\text { investigadora }\end{array}$ & $\begin{array}{l}\text { Tipo de documento, número } \\
\text { de página y párrafo. }\end{array}$ & $\begin{array}{l}\text { DI.54.3 (Diario de campo, número de } \\
\text { página, párrafo 3). }\end{array}$ \\
\hline $\begin{array}{l}\text { Informes de la } \\
\text { investigación }\end{array}$ & $\begin{array}{l}\text { Tipo de documento, número de } \\
\text { Informe, página } \\
\text { y párrafo. }\end{array}$ & $\begin{array}{l}\text { I3.4.2 (Informe número 3, página } \\
\text { 4, párrafo 2). }\end{array}$ \\
\hline $\begin{array}{l}\text { Producciones } \\
\text { del alumnado }\end{array}$ & $\begin{array}{l}\text { Tipo de documento, fecha, número } \\
\text { de lista. }\end{array}$ & $\begin{array}{l}\text { P.16/02/18.17 (Producción de alumno. } \\
16 \text { febr 2018. Número } 17 \text { de la lista } \\
\text { alumnado). }\end{array}$ \\
\hline $\begin{array}{l}\text { Evaluación de la tutora } \\
\text { y Mtra. de biblioteca }\end{array}$ & Tipo de documento, página y párrafo. & $\begin{array}{l}\text { AT.1.6 (Análisis de la tutora del grupo, } \\
\text { página 1, párrafo 6). }\end{array}$ \\
\hline Entrevistas & $\begin{array}{l}\text { Tipo de documento, número de } \\
\text { entrevistado, número de pregunta. }\end{array}$ & $\begin{array}{l}\text { E.6.8 (Entrevista, entrevistado número } \\
\text { 6, pregunta 8). }\end{array}$ \\
\hline
\end{tabular}

\section{Resultados y Discusión}

En el proceso de implementación de LÓVA, durante el curso escolar 2017-18, se observó una notable mejora en el desarrollo de la competencia ciudadana del grupo de alumnos de Educación Primaria del "Instituto Luis Vives" (que conformó este estudio de caso). Interesante fue la mejora de algunos niños que anteriormente mostraban conductas disruptivas y que, con el proyecto LÓVA, lograron integrarse al grupo de forma satisfactoria. Tal y como refrendan Bradberry y Graves (2009) y Goleman (1995), la inteligencia emocional es la capacidad de sentir, entender, controlar y hasta modificar los estados de ánimo tanto propios como ajenos: "Hemos aprendido más de nuestros compañeros y ahora nos entendemos mejor" 


\section{(P.11/12/17.14), comentó un estudiante.}

Para la selección del grupo que conformaría el estudio de caso se tuvo en cuenta que el salón contaba, como ya se indicó, con algunos casos de conductas disruptivas, con dos estudiantes con timidez aguda e inseguridad frente al grupo y con otros, previamente diagnosticados, con mutismo selectivo, Asperger y diabetes infantil. Se trataba de un salón que no había conseguido consolidarse como grupo hasta ese momento y rara vez se habían observado conductas de compañerismo. En ese sentido cobra importancia la propuesta de Rose (2009) de incluir en el currículum de la Primaria a las artes escénicas como vía para la mejora en habilidades emocionales y competencias para el desarrollo de la ciudadanía.

\subsection{Derechos y responsabilidades}

Las competencias ciudadanas son un conjunto de conocimientos, actitudes y habilidades de carácter cognitivo, emocional y comunicativo. Todas ellas, articuladas entre sí, hacen que el ciudadano democrático esté dispuesto a actuar de manera constructiva y justa en la sociedad (Muñoz y Vergara, 2015). Las votaciones se establecieron como mecanismo de decisión al interior de la compañía. Esta herramienta democrática sirvió tanto para la elección del nombre de la compañía como para la decisión del logotipo que la representaría, por citar dos ejemplos. También resulta importante referir que los alumnos detectaron una irregularidad en el proceso de la primera votación para la elección de su logotipo. Inmediatamente y de forma unánime se tomó la decisión de invalidarla y repetirla de nuevo (12.8.2).

La práctica y ejercicio de la ciudadanía propicia el desarrollo de actitudes y destrezas que se requieren para convertirse en un miembro activo, crítico pero responsable y reflexivo de un pueblo que pretende la organización política democrática para la comunidad (Villarini, 1987). También se trabajó el conocimiento de derechos y responsabilidades a través de, entre otras actividades, una leyenda africana llamada "Olelé" -que simboliza el trabajo colaborativo y el conocimiento de los derechos y responsabilidades de cada integrante de un poblado.

Hemos aprendido muchas cosas y es que a nosotros se nos movió la tierra el pasado 19 de septiembre, ahí donde hacemos las reuniones de la compañía de ópera, y ya después nada fue igual. Por eso, la compañía no quería dejar a nadie detrás, que no estuviera de acuerdo... los árboles no se cayeron cuando la tierra se movió debajo de nuestros pies, allí donde nos sentíamos seguros, en nuestra escuela, en nuestro salón... (11.11.1).

Cuando a los alumnos se les preguntó el porqué del nombre de la compañía de ópera (La hoja del árbol), dijeron que se sentían como hojas que conformaban un árbol, tan fuerte que pudo resistir al temblor referido. Esta reflexión fue recogida en el diario de investigación. Cabe recordar que los espacios de educación musical ayudan a propagar valores culturales, construyen el mundo social en el que operan las identidades de los niños (Bennett, 2000; Walker, 2007) y generan la cohesión social necesaria para el desarrollo de la competencia ciudadana (Zambrano, 2018): "Ha adquirido una conciencia de pertenencia al saberse un elemento valioso de los grupos con los que interactúa, incluida la familia. Su capacidad de interrelacionarse ha mejorado" (E.14.3), opinaba un padre al finalizar el proyecto LóVA.

En algunas definiciones de competencia ciudadana encontramos la capacidad para participar en múltiples contextos, ámbitos sociales y laborales en los que se desempeña el individuo (Carrillo, 2013). "Se volvió muy independiente y segura de sus decisiones. Aprendió también el valor y la importancia de ser parte de un grupo y de tomar en cuenta sus decisiones para poder avanzar" (E.3.2). Es otra de las apreciaciones al concluir LÓVA. En efecto, la formación ciudadana también involucra la educación de los derechos humanos para constituirse en el ejercicio de estos. En la experiencia como ser social, el estudiante se asume como propio constructor de su entorno y empieza a empoderarse en diversos ámbitos. El fin último de la educación para la ciudadanía debe perseguir la educación para la democracia y la construcción de contextos que propicien la aplicación de principios y rutinas. Los alumnos deben facultarse igualmente para que lleguen a ser miembros de una comunidad con vistas a participar y desempeñar papeles articulados en el espacio público (Riddle y Apple, 2019). 
"Se interesa en lo que pasa en la ciudad o en el país, escucha noticias y las comenta. Platica también sobre sus compañeros y cómo se sienten en la escuela" (E.11.7); "reconoce que hay responsabilidades y que debe cumplirlas" (E.11.8), son otros comentarios de las familias sobre la adquisición de responsabilidades a través del proyecto LÓVA.

Hacia el final del proyecto, la reflexión en cuanto a la mejora en el reconocimiento de derechos y responsabilidades de una de las maestras fue la siguiente: "Están siendo muy responsables tanto con el proyecto LÓVA como con sus trabajos diarios escolares y cumplen con todas sus obligaciones" (AT.2.2). Los alumnos deben lograr ser capaces de exigir sus derechos para asumir sus propias responsabilidades al mismo tiempo que pueden vivir con otros según los valores democráticos (Escudero y Flecha, 2005). En la construcción del carácter del ciudadano personalmente responsable se enfatiza la honestidad, la autodisciplina, la integridad y el trabajo constante (Westheimer, 2020).

Una última acotación a la relevancia del compromiso y la adquisición de responsabilidades se encuentra en el siguiente testimonio: "Su voz aguda revela que es un niño. Su lenguaje elaborado, lo increíble: es director de Relaciones Públicas. No es ningún juego. (J) se toma su trabajo con la mayor seriedad. A sus 10 años es jefe de prensa de "La hoja del árbol", una compañía de ópera que fundó en septiembre pasado con sus 19 compañeros del Instituto Luis Vives" (Bautista, 2018).

\subsection{Compromiso y participación social}

El temblor del 19 de septiembre de 2017 interrumpió la rutina de las reuniones de la compañía de ópera y, al retomar las actividades escolares, el proyecto se convirtió en un espacio de reflexión personal y grupal de las experiencias vividas. "Lo que hicimos para ayudar en el temblor... yo fui a dos centros de acopio, aunque yo hubiera querido ayudar a quitar escombros, pero no pude porque soy pequeño" (P. 6/10/17.9), comentó un alumno que pensó que no lo dejaron ayudar lo suficiente. Todos los miembros del grupo expusieron las experiencias vividas durante y después del temblor y cada estudiante definió en una palabra el sentimiento que le provocó. Se hizo un mural en el salón con todos esos sentimientos y el grupo decidió que se quedaría expuesto hasta que se superaran los sentimientos anotados (11.3.1).

En el escrito acerca del sismo y de los días que le sucedieron se observó que los niños muestran gran solidaridad y desean ayudar a las personas que han perdido casa, mascotas o seres queridos. Muchos de ellos ayudaron entonces llevando ropa, juguetes, medicamentos a los campamentos de acopio y sintieron que aportaban su granito de arena haciendo que otros niños y adultos se sientieran mejor (11.7.2).

Me acuerdo del temblor, de los que perdieron sus casas, sus hijos o sus vidas... me entristece mucho porque me siento como si yo las hubiera perdido, además yo cooperé en un centro de acopio para pequeños enfrente de mi casa y empaqueté toda la ropa para bebés y les puse nombre... sentí que ayudaba a las personas sin hogar (P. 6/10/17.12).

El sentimiento y compromiso del alumnado también quedó plasmado en la siguiente cita que relaciona directamente el trabajo en la compañía de ópera con el reto de hacer y estrenar una ópera original desde cero sin ayuda de adultos: "Valor... y pensé... tenemos que ir adelante y no nos rendiremos, podemos hacerlo en equipo, no nos vamos a rendir" (P.6/10/17.15). El ciudadano responsable y participativo "take part in the civic affairs and social life of the community al local, state/provincial, national, and sometimes global levels" (Westheimer, 2020, p.9). Cooperar para aprender supone la superación del aprendizaje individual en favor del colectivo y reconocer que con los compañeros se pueden llevar a buen término tareas que individualmente resultarían imposibles de realizar (Cangro, 2015).

El desarrollo del compromiso y participación social a partir del autoconocimiento y de la posibilidad de adueñarse de las situaciones en las que el estudiante se desenvuelve, no sólo se queda en el aula, sino que, como afirman Kincheloe y McLaren (2012), los niños pueden y deben crear una conciencia social que sea susceptible de transformar la realidad. La educación no dota de herramientas a las personas para ejecutar tareas específicas, sino que las empodera para transformarlas, para juzgar cuándo y si son apropiadas, e incluso para rechazar hechos que han sido negados de forma irrefutable (Bowman, 2002). 
En la compañía de ópera infantil "La hoja del árbol" se establecieron y desarrollaron las relaciones sociales, se mejoró la autoconfianza grupal e individual y se avanzó en la adquisición de la competencia interpersonal. El concepto de trabajo colaborativo se extiende en esta investigación a cualquier tarea que requiera la participación y el compromiso de un mínimo de dos alumnos. De igual manera, por desarrollo social se entiende la mejora en el conjunto de habilidades que se suceden en el comportamiento grupal y en las interacciones con el otro (Baker y Harvey, 2014). La investigación permitió comprobar cómo los alumnos participantes en el proyecto LóVA desarrollaron su autoconfianza y autoestima. Se logró empoderar a los miembros de la compañía de ópera infantil al conferirles seguridad en sí mismos. El gran logro en el ámbito de la relación interpersonal consistió en que los estudiantes se articularan como un grupo unido y con un fuerte sentimiento de pertenencia a la institución. La compañía supo consolidarse como un grupo único con un objetivo común: el estreno de la ópera.

El compromiso ahora ya es total y los logros son de todos los miembros, el individualismo quedó atrás cuando se trata de celebrar alguna buena noticia de la compañía: Podemos poner el ejemplo del día que resolvimos toda la historia: (14.19.1)

Son muchos los que proponen ideas y cómo debe ser el desenlace. Decidimos que se juntarán las ideas para organizar toda la tercera escena con el final incluido, en medio del caos y ruido que se escucha en la escuela, demostrando mucha tolerancia y compromiso con nuestro trabajo y la comunidad en general. (14.19.2).

\subsection{Respeto y valoración de la diversidad}

La formación ciudadana pretende formar personas empáticas interesadas en el bienestar común con autonomía moral. Los alumnos deben aprender a construir con otras personas un orden social capaz de mejorar las formas de relación para contribuir a una vida digna para todos (Conde, 2016).

Durante el desarrollo de LÓVA y a propuesta de los sujetos participantes en el estudio de caso, surgió la necesidad de mantener charlas acerca del bullying. Las preguntas que detonaron la reflexión fueron: ¿por qué molestamos u ofendemos a los compañeros? ¿cuáles son los motivos? A partir de estos cuestionamientos resultó necesario considerar las bromas como forma de acoso. Todos los miembros de la compañía opinaron y fueron escuchados. Se profundizó en el bullying físico y psicológico y fueron ellos mismos quienes propusieron un ejercicio de valentía en el que cada alumno expresaba qué le molestaba en el comportamiento y en el trato de los demás compañeros. Apodos, comparaciones, y situaciones más graves como ataques con violencia física por otros compañeros, fueron expuestos en estos ejercicios (I3.12.1).

Se aprovechó la oportunidad para hablar de las diferencias entre las personas y para considerar la diversidad como una riqueza, al tiempo que se reflexionó sobre cómo precisamente esas diferencias pueden resultar idóneas para que la comunidad funcione. Finalmente, se entendió la compañía de ópera como paradigma de diversidad (13.11.3). Banks (2017) propone que el reconocimiento de la "ciudadanía diversa" se logra con la participación efectiva en la comunidad. La idea de la escuela como comunidad que se vincula con el sentido de pertenencia y responsabilidad compartida se materializa en la institución que comparte valores como el respeto, el diálogo, el trabajo colaborativo y el valor de la diferencia (Rojas y Haya, 2018).

Travis et al. (2019) comprobaron que los ejercicios sobre el autoconocimiento benefician de igual manera a la autoestima y a la autoeficacia. La autoestima no sólo se refiere a la percepción de uno mismo sino también al ideal que se tiene de uno mismo, mientras que la autoeficacia se relaciona directamente con la habilidad de tener un impacto en el mundo que nos rodea. Además, la adquisición de confianza en un contexto cultural aceptado por el alumnado contribuye a la formación del capital social. Se recogen aquí algunos de los mensajes que los niños dejaron para sus compañeros en el mural de frases: 
Mensaje para alguien que sufra bullying: no se den por vencidos, ustedes son únicos y no son ninguna cosa o persona fea, mala para el mundo, ni tonta. Su potencial no se acaba ni su inteligencia, aprovéchenla... no respondan con violencia y resuelvan todo hablando (P.08/02/18.13).

Tienes que ponerte en su lugar para entender que tienes que ayudar porque es muy feo ver que le están haciendo eso, y también te imaginas que te hagan eso a ti... no me gustaría que los demás sufran lo mismo que yo (P.08/02/18.12).

Conde (2016) comenta que la educación para la ciudadanía debe formar alumnos reconocedores del otro, que respeten la diversidad a la vez que defiendan la igualdad de género, la multiculturalidad y cualquier forma de pluralidad. Como ejemplo, en nuestra compañía infantil de ópera se llegó al acuerdo por unanimidad de no responder con violencia a las agresiones provocadas por un integrante del grupo. En su lugar se le respondió con compañerismo, amistad, respeto y cariño (2/2/18). Por otro lado, las familias opinaron que "obviamente su capacidad de trabajo colaborativo y capacidad de llegar a acuerdos, delegar responsabilidades, crear alianzas y respetar la diferencia de opiniones fue algo a lo que se vio sometida durante este proceso de aprendizaje" (E.16.3).

La formación para la ciudadanía democrática procura alcanzar los fines propuestos mediante la educación en derechos humanos, valores y en la educación para la construcción de la democracia (Osler, 2018). Por otro lado, se requiere de una gestión de la diversidad que esté basada en la planificación de la enseñanza en función de la diversidad del alumnado y sus necesidades (Bergeron, 2015). "Se hizo más abierta respecto a las múltiples maneras de pensar y de actuar, entendió mejor que la gente tiene diferentes habilidades, fortalezas y perspectivas" (E.16.4), comentaba un familiar sobre la adquisición de la competencia ciudadana, "pero ahora sabe que puede ser parte de cualquier grupo sin tener prejuicios tanto para con ella como para con los demás" (E.1.5), "aprendió que todos son distintos y tienen diferentes capacidades y aptitudes para diferentes cosas" (E.7.5). Son algunas de las respuestas sobre reconocimiento a la diversidad y respeto a la diferencia que dieron las familias en la entrevista al final del proyecto.

Durante la última etapa de la implementación de LÓVA se observó cómo el aprendizaje grupal de algunos valores éticos como el respeto, lealtad, honestidad y equidad mejoraron para toda la compañía (14.17.1). En su nota periodística, Piñón (2018) hace referencia al clima que se respira al interior del salón durante las reuniones de la compañía "La hoja del árbol", señala: "Los niños caminan con seguridad, sonríen, bromean, se abrazan a la menor provocación, se percibe entre ellos la amistad, la camaradería, el respeto".

Durante la visita del supervisor de la Secretaría de Educación Pública (SEP), la compañía se presentó y respondió a las preguntas que se le formularon sobre el proyecto. Cada grupo explicó las profesiones, su propuesta de ópera y en qué consistía el proyecto en general. El supervisor hizo algunas preguntas referentes al proyecto y a la luz de las respuestas les felicitó por la ópera, su compromiso y el grado de madurez encontrada:

Son un equipo y es muy importante lo que dicen que han aprendido: tomar acuerdos, compañerismo, empatía, igualdad, equidad, todos somos importantes. Valores como el respeto, la convivencia, la empatía. El razonamiento profundo, conocerse mejor. ¡No hay bullying! (Prof. Juan Martín Vigueras, supervisor de zona núm. 128 de la SEP en la CDMX) (I.5.7.1).

Para Apple (2017) la educación es un acto político y ético y requiere que la situemos en las relaciones que se establecen en la sociedad. A este respecto se refería un familiar entrevistado: "Noté que utilizaba un lenguaje muy solidario al comentar los obstáculos que repentinamente llegaban a enfrentar, tanto los compañeros de su equipo como los de otros equipos" (E.21.5).

En las últimas tres décadas la globalización y la educación para la ciudadanía se han vuelto conceptos clave en las Ciencias Sociales. La participación de la pedagogía crítica se hace necesaria en la construcción de lo que significa la ciudadanía global y el significado de los derechos y responsabilidades que conlleva (Freire, 2008). Por consiguiente, las prácticas artísticas en proyectos escolares necesitan revisar esa concepción de ciudadanía y la responsabilidad que se requiere para poderla poner en práctica (Elliott et al., 2016). 
Aprendió la importancia de mantener un grupo equilibrado, unido, lo que es trabajar en equipo para lograr un objetivo en común y para él fue muy significativo observar que la "igualdad" es lo que los hace similares pero el "respeto" lo que los hace diferentes y así poder convivir en armonía (E.7.9).

\section{Conclusiones}

La implementación del proyecto LóVA demostró que es un recurso eficaz para el desarrollo de la competencia cívica tanto a nivel individual como grupal. Se propusieron los siguientes ámbitos: 1) la colaboración para aprender de los demás, el respeto a sus necesidades, la empatía y el ser sensible a la hora de abordar conflictos para así facilitar la resolución de problemas de manera participativa y colaborativa; 2) se desarrolló el pensamiento crítico, el cuestionamiento de las prácticas, normas y diversas opiniones, la reflexión sobre las acciones propias y la disposición de una opinión fundada en los diferentes temas en tanto que ciudadano responsable y activo; 3 l la autoconciencia, la reflexión sobre el papel de cada uno en la sociedad, al mismo tiempo que la necesidad de conocer y combatir los sentimientos propios y deseos personales, y 4) se abordó la resolución alternativa de conflictos: idear y aplicar diferentes soluciones a los problemas de sostenibilidad para obtener soluciones equitativas que fomentan el desarrollo armónico a través de las subcompetencias arriba mencionadas.

Bradley (2018) insiste precisamente en que el reto de los docentes musicales consiste en promover en el alumnado el desarrollo de la responsabilidad social, la preocupación por el otro, el cuidado del medio ambiente y el rechazo absoluto al racismo y heterosexismo a través de la expresión artística. Por otro lado, el desarrollo de LÓVA, además de atender a la educación estética y emocional por los motivos referidos antes, conlleva profundas implicaciones en lo que respecta al aprendizaje moral. La resolución de conflictos, la empatía, el respeto, el reconocimiento a la diversidad, el compromiso social y la participación en la comunidad y conocimiento de derechos y responsabilidades sitúan las competencias de este proyecto no sólo en el ámbito artístico y científico sino en el territorio de lo ético-social.

La transformación política y la transmisión cultural en forma de creencias, valores y actitudes han influenciado este nuevo patrón de educación para la ciudadanía a través de la educación musical en la era de la globalización (Ho, 2019). Se ha visto cómo la música provee a los niños de incontables posibilidades de regulación en sus estados de ánimo y posibilita la mejora de la atención, de la motivación y la creatividad (Joy, 2002), al mismo tiempo permite la conexión entre las personas y el manejo de las emociones propias (Baltazar y Saarikallio, 2017; Groarke y Hogan, 2015).

En LÓVA encontramos un proyecto para la formación de la ciudadanía global a través del aprendizaje artístico y la educación emocional. Elliott (2012) propone una educación musical guiada éticamente para pensar la música, de esta manera se puede empoderar a los alumnos "to pursue what many philosophers throughout history consider to be the highest human values: a virtuous life well lived, a life of well-being" ( $p$. 22). El concepto de ciudadanía global implica el autoconocimiento y la relación con la sociedad y la cultura para contribuir productivamente a la comunidad. La empatía se define ahora en el conocimiento de otras culturas y personas, a través de una formación que implique descubrimiento y concientización de eventos y situaciones en otras partes del mundo (Grant, 2018).

El reforzamiento de las competencias sociales y cívicas en el alumnado participante en LÓvA incluye habilidades personales, interpersonales y de ciudadanía; en ellas encontramos formas de comportamiento que los prepararán para participar de manera eficiente y constructiva en la vida en comunidad, especialmente en sociedades cada vez más diversas (Giraldez-Hayes et al., 2019). Conviene recordar que la meta 4.7 de los ODS (ONU, 2015) contempla que para el 2030 se debe garantizar que todos los niños adopten un estilo de vida sostenible, se promueva en ellos la igualdad de género, los derechos humanos, una cultura de paz y no violencia, la ciudadanía global y la valoración de la diversidad cultural.

En definitiva, la implementación de LÓVA contribuyó decididamente al desarrollo de una conciencia social y favoreció la adquisición de responsabilidad desde lo local hacia lo global, a través de la expresión artística mediante la creación de una ópera original. 


\section{Referencias}

Acevedo, R. A. y López, P. (2012). Ciudadanos inesperados: espacios de formación de la ciudadanía ayer y hoy. El Colegio de México/Centro de Investigación y de Estudios Avanzados.

Apple, W. M. (2017). The struggle for democracy in critical education. Revista e-Curriculum, 15(4), 894-924.

Baker, W. J. y Harvey, G. (2014). The collaborative learning behaviours of middle primary school students in a classroom music creation activity. Australian Journal of Music Education, 1, 3-14.

Baltazar, M. y Saarikallio, S. (2017). Strategies and mechanisms in musical affect self-regulation: A new model. Musicae Scientiae, (1-19). https://doi.org/10.1177/1029864917715061

Banco Interamericano de Desarrollo. (2011). Informe Nacional de Resultados. México. Estudio Internacional de Educación Cívica y Ciudadana, ICCS, 2009. UNESCO-CERLALC, SREDECC.

Banks, J. A. (2017). Failed citizenship and transformative civic education. Educational Researcher, 46(7), 366377. https://doi.org/10.3102/0013189X17726741

Bautista, E. (2018, 15 de junio). Ópera para el recreo. El Financiero.

https://www.elfinanciero.com.mx/culturas/opera-para-el-recreo

Bennett, A. (2000). Popular music and youth culture: Music, identity and place. Macmillan.

Bergeron, L. (2015). La planification de l'enseignement a priori en fonction de la diversité des élèves. Une logique préventive et proactive [La planificación de la docencia a priori según la diversidad de los alumnos. Una lógica preventiva y proactiva]. En N. Rosseau (Dir.), La pédagogie de l'inclusion scolaire: Un défi ambitieux et stimulant (pp. 375-397). Presses de l’Université du Québec.

Bohnsack, R. (2004). Group discussions and focus groups. En U. Flick, E. Kardoff e I. Steinke (Eds.), A companion to qualitative research (pp. 214-221). Sage.

Bowman, W. D. (2002). Educating musically. En R. Colwell y C. Richardson (Eds.), The new handbook of research on music teaching and learning (pp. 63-84). Oxford University Press.

Bradberry, T. y Greaves, J. (2009). Emotional intelligence 2.0. Talent Smart.

Bradley, D. (2018). Artistic citizenship: Escaping the violence of the normative(?). Action, Criticism, and Theory for Music Education, 17(2),71-91. http://act.maydaygroup.org/volume-17-issue-2/act-17-2-71-91/

Cabedo-Mas, A., Nethsinghe, R. y Forrest, D. (2017). The role of the arts in education for peacebuilding, diversity and intercultural understanding: A comparative study of educational policies in Australia and Spain. International Journal of Education \& the Arts, 18(11). http://www.ijea.org/v18n11/

Cangro, R. (2015). Student collaboration and standards-based music learning. Update: Applications of Research in Music Education, 34(3), 63-68. https://doi.org/10.1177/8755123314568794

Carrillo, O. (2013). Comprendiendo la adquisición de las competencias ciudadanas en alumnos de los programas de cualificación profesional inicial. Revista Educar, 49(2), 207-226.

Conde, L. S. (2016). Formación ciudadana en México. Instituto Nacional Electoral.

Cox, C. (2010). Informe de referente regional 2010. Oportunidades de aprendizaje escolar de la ciudadanía en América Latina: currículos comparados. SREDECC, Pontificia Universidad Católica de Chile. 
Delors, J. (1996). Learning: The treasure within. UNESCO.

Díaz, E. (2015). Diversidad lingüística y ciudadanía en educación. En E. Díaz et al. (Coords.), Diversidad, ciudadanía y educación. Sujetos y contextos (pp. 49-75). Universidad Pedagógica Nacional.

Dullea, R. (2017). Engagement, participation, and situated learning in a children's opera chorus program. Journal of Research in Music Education, 65(1), 72-94. https://doi.org/10.1177/0022429417695751

Dullea, R. (2019). Pedagogies of authentic learning in Canadian Opera Company's opera summer camp programme for adolescents. Youth Theatre Journal, (1-17).

https://doi.org/10.1080/08929092.2018.1555103

Elliott, D. J. (2012). Another Perspective. Music Educators Journal, 99(1), 21-27.

https://doi.org/10.1177/0027432112452999

Elliott, D., Silverman, M. y Bowman, W. (Eds). (2016). Artistic citizenship: Artistry, social responsibility, and ethical praxis. Oxford University Press.

Escudero, J. M. y Flecha, R. (2005). Ciudadanía y democracia: el núcleo central de una buena educación para todos. En Proyecto Atlántida. Ciudadanía, mucho más que una asignatura. Ministerio de Educación y Ciencias y Gobierno de Canarias.

Faust, C. (2010). BRAVA! Why you should use Opera in your classroom. Education Digest: Essential Readings Condensed for Quick Review, 76(4), 55-58.

Flores-Crespo, P. y Ramírez, A. V. (2015). La participación social en la escuela de México. Una revisión de la literatura. Revista Iberoamericana sobre Calidad, Eficacia y Cambio en Educación, 13(3), 77-94.

Freire, P. (2008). Cartas a Cristina. Reflexiones sobre mi vida y mi trabajo. Siglo XXI editores.

Giraldez-Hayes, A., Carbó, G. y Otárola, M. (2019). Evaluación proyecto LÓVA. La ópera, un vehículo de aprendizaje 2006-2017. ConArte Internacional \& Fundation Daniel y Nina Carasso.

Giroux, H. (2013). La pedagogía crítica en tiempos oscuros. Praxis Educativa, $17(1$ y 2), 13-26.

Goleman, D. (1995). Emotional Intelligence. Michigan University-Bantman Books.

Grant, C. (2018). Developing global citizenship in tertiary performing arts students through short-term mobility programs. International Journal of Education \& the Arts, 19(15).

https://doi.org/10.18113/P8ijea1915

Groarke, J. M. y Hogan, M. (2015). Enhancing wellbeing. An emerging model of the adaptative funtions of music listening. Psychology of Music, 1-23. https://doi.org/10.1177/0305735615591844

Higgins, L. (2015). Hospitable music making: Community music as a site for social justice. En C. Benedict, P. Schmidt, G. Spruce, y P. Woodford (Eds.), The Oxford Handbook of Social Justice in Music Education (pp. 446455). Oxford University Press.

Ho, W. C. (2019). Citizenship education and music education: Exploring the crossroads between globalisation and localisation in Taiwan. Music Education Research, 1-15.

https://doi.org/10.1080/14613808.2019.1598347

Joy, S. (2002). Original student opera: History, theory, and practice of a multi-disciplinary arts education program [Tesis Doctoral, University of Houston]. 
Kincheloe, J. L. y McLaren, P. (2012). Replanteo de la teoría crítica y de la investigación cualitativa. En N. K. Denzin y Y. S. Lincoln (Eds.), Manual de investigación cualitativa. Paradigmas y perspectivas en disputa (pp. 241-315). Gedisa.

Muñoz, D. A. y Vergara, L. M. (2015). Educar para la paz justa y duradera: una visión desde Macintyre. En J. C. Arboleda (Ed.) Innovaciones y Educación para la paz. Simposio Internacional de Educación y Pedagogía (pp. 642-655). REDIPE.

Nieto, D. y Bickmore, K. (2016). Educación ciudadana y convivencia en contextos de violencia: desafíos transnacionales a la construcción de paz en escuelas de México. Revista Española de Educación Comparada, 28, 109-134.

ONU (2015). Educación para los Objetivos de Desarrollo Sostenible. Objetivos de Aprendizaje. Autor.

Osler, A. (2018). Human rights education: a project for our common future. Human Rights Education Review, 1(1), 1-4. https://doi.org/10.7577/hrer.2805

Piñón, A. (30 de marzo de 2018). La ópera también es cosa de niños. El Universal. https://www.eluniversal.com.mx/cultura/la-opera-tambien-es-cosa-de-ninos

Riddle, S. y Apple, M. W. (Eds.). (2019). Re-imagining education for democracy. Routledge.

Rojas, S. y Haya, I. (2018). Fundamentos pedagógicos de atención a la diversidad. Universidad de Cantabria.

Rose, J. Sir. (2009). The independent review of the primary curriculum: Final report. Department for Children, Schools and Families.

Saint Paul, J. (2011). TH Marshall y las discusiones contemporáneas sobre ciudadanía, cohesión social y democracia. Estudios Políticos, Documento de Trabajo, 1. Serie Estudios Políticos. Universidad de Guanajuato.

Sarmiento, P. (2012). La ópera, un vehículo de aprendizaje (LÓVA). Eufonía. Didáctica de la Música, 55, 4047.

Simons, H. (2011). El estudio de caso: teoría y práctica. Morata.

Stake, R. E. (2005). Qualitative case studies. En N. K. Denzin y Y. S. Lincoln (Eds.), The SAGE handbook of qualitative research (pp. 443-465). Sage.

Starkey, H. (2017). Globalization and education for cosmopolitan citizenship. En J. A. Banks (Ed.), Citizenship and global migration: Implications for theory, research, and teaching (pp. 41-61). American Educational Research Association.

Tonucci, F. (2017). A modo de Introducción... La diversidad como valor en una escuela que cambia. Aula Abierta, 46, 9-12.

Travis, R., Gann, E., Crooke, A. H. D. y Jenkins, S. M. (2019). Hip Hop, empowerment, and therapeutic beatmaking: Potential solutions for summer learning loss, depression, and anxiety in youth. Journal of Human Behavior in the Social Environment, 1-22.

Villarini, A. (1987). Principios para la integración del currículo. Departamento de Instrucción Pública.

Walker, R. (2007). Music education: cultural values, social change and innovation. Charles C. Thomas. 
Westheimer, J. (2020). Can education transform the world?, Kappa Delta Pi Record, 56(1), 6-12.

https://doi.org/10.1080/00228958.2020.1696085

Zambrano, E. L. (2018). Prácticas pedagógicas para el desarrollo de competencias ciudadanas. Revista Electrónica de Investigación Educativa, 20(1),69-82. https://doi.org/10.24320/redie.2018.20.1.1409

Zurita, U. (2010). La educación para la vida democrática a través de la participación social: puntos de encuentro entre la escuela y la familia. Revista Interamericana de Educación para la Democracia, 3(2), 51-74. 